\title{
Variability of Distal Femoral Valgus Resection Angle in Patients with End-stage Osteoarthritis and Genu Varum Deformity: Radiographic Study in An Ethnic Asian Population
}

\author{
Chien-Yin Lee ${ }^{1}$, Tsan-Wen Huang ${ }^{1}$, Kuo-Ti Peng ${ }^{1}$, Mel S. Lee ${ }^{1}$, Robert Wen-Wei Hsu \\ Wun-Jer Shen ${ }^{2}$
}

Background: When performing a total knee arthroplasty, most surgeons use the intramedullary alignment guide with a fixed distal femoral valgus resection angle. In this study, we assessed the variability of the distal femoral valgus resection angle in ethnic Asian patients by reviewing our arthroplasty database.

Methods: $\quad$ Between January 2004 and December 2012, the patients with end-stage osteoarthritis with genu varum deformity who underwent total knee arthroplasty were enrolled in this retrospective review. Clinical and radiographic data were collected and analyzed.

Results: $\quad$ Nine hundred and fifty-two knees met the inclusion criteria. Three hundred and four (31.9\%) knees had a distal femoral valgus resection angle value outside the range of $5^{\circ} \pm 2^{\circ}\left(\right.$ range, $\left.4^{\circ}-14^{\circ}\right)$. There were significant differences in the mean distal femoral valgus resection angle between males and females $(p<0.001)$ and between non-bowed femur and bowed femur $(p<0.001)$ cohorts. With regard to the correlation coefficients between the distal femoral valgus resection angle and the usual radiographic measurements, only the coronal

\section{At a Glance Commentary}

Scientific background of the subject

The distal femoral valgus resection (DFVR) angle is highly variable and leads the intramedullary guide unreliable, but scant data specific for ethnic Asians has been reported in the literature. The aim of this study was to determine the variability in ethnic Asian patients with end-stage osteoarthritis and genu varum deformity.

\section{What this study adds to the field}

$32 \%$ of ethnic Asians patients have a DFVR angle that is outside the range of $5^{\circ} \pm 2^{\circ}$. Special jigs that allow a wide enough choice of valgus cut angle, simultaneous or staged corrective osteotomy combined with TKA, or intra-articular bone resection technique can be used to accommodate the deformity and provide ideal reconstructed alignments. femoral bowing angle demonstrated a good correlation $(r=0.72)$.

Conclusions: $32 \%$ of Asian patients present with a distal femoral valgus resection angle that is outside the range of $5^{\circ} \pm 2^{\circ}$. Taking a long-leg weight-bearing split scanogram may provide information that allows the surgeon to determine the true distal femoral valgus resection angle and adjust the cut accordingly.

Level of Therapeutic level III.

Evidence:

(Biomed J 2015;38:350-355)

Key words: distal femoral valgus resection angle, end-stage osteoarthritis, ethnic Asian population, total knee arthroplasty

From the ${ }^{1}$ Department of Orthopaedic Surgery, Chang Gung Memorial Hospital at Chiayi, Chang Gung University College of Medicine, Taoyuan, Taiwan; ${ }^{2}$ Department of Orthopaedic Surgery, Po-Cheng Orthopaedic Institute, Kaohsiung, Taiwan Received: Jun. 25, 2014; Accepted: Jan. 13, 2015

Correspondence to: Dr. Tsan-Wen Huang, Department of Orthopaedic Surgery, Chang Gung Memorial Hospital at Chiayi. 6, West Section, Chia-Pu Rd, Pu-Tz, Chiayi 613, Taiwan (R.O.C.). Tel: 886-5-3621000 ext. 2855; Fax: 886-5-3622188;

E-mail: b8601081@adm.cgmh.org.tw

DOI: $10.4103 / 2319-4170.151030$ 
L ong-term survival analysis has shown that the longevity of the implants and optimal long-term outcomes depend on the accuracy of bone cuts and the proper restoration of the mechanical axis of the leg. ${ }^{[1-9]}$ An intramedullary (IM) alignment guide is currently the most commonly used method for performing the distal femoral resection in total knee arthroplasty (TKA). Finite element models confirm that bone-cut errors should be perpendicular to the femoral mechanical axis to prevent abnormal wear, premature mechanical loosening of the components, and patellofemoral problems. ${ }^{[10]}$ In practice, most surgeons use a fixed $5^{\circ}-6^{\circ}$ resection angle relative to the IM rod for patients with preoperative genu varum deformities, ${ }^{[11-15]}$ but postoperative malalignment of the femoral component of greater than $3^{\circ}$ occurs in up to $20 \%$ of cases when using an IM guide. ${ }^{[6,16-19]}$

Malorientation of the femoral component relative to the femoral axis has been shown to significantly increase the aseptic failure rate. ${ }^{[20]}$ There are several causes of femoral component malalignment when an IM alignment guide is used. First, the IM guides rely on a proper fit in the IM canal. Second, the position of the guide is affected by the position of the entrance hole through the distal femur into the IM canal. As a result, a properly restored mechanical axis is often difficult to achieve in the presence of diaphyseal deformity, distortion of the osseous canal, residual implants from previous surgery, malunited fractures, metabolic bone disease, and variations in femoral anatomy such as a large IM canal and excessive femoral bowing. ${ }^{[12,21-27]}$ In the majority of TKA systems, the design of the IM cutting guides assumes that the difference between the femoral mechanical and anatomical axes is approximately $5^{\circ} .{ }^{[21]}$ It is known that the angle between the femoral mechanical axis and anatomical axis is highly variable and affected by the patient's age, gender, body height, neck-shaft angle, and femoral offset. ${ }^{[15,28,29]}$ However, we are aware of little data reported on the preoperative demographic and radiographic variables specific for ethnic Asians in the literature. The aim of this study was to determine the variability of the distal femoral valgus resection angle in ethnic Asians patients with end-stage osteoarthritis and genu varum deformity.

\section{METHODS}

This study was approved by the Institutional Review Board at the Chang Gung Memorial Hospital (98-1038B) and comprised a total of 526 patients who underwent TKA at the hospital between January 2004 and December 2012. The inclusion criteria were patients suffering from end-stage osteoarthritis with genu varum deformity and who underwent a primary TKA. The exclusion criteria included patients with a history of prior hip arthroplasty with endoprosthesis replacement, developmental dysplasia of the hip, prior fracture of femur or tibia, or incomplete medical records with respect to radiographic analyses.

All patients were evaluated preoperatively using radiographic analyses with anteroposterior (AP) and lateral radiographs of the knees. Long-leg weight-bearing split scanograms previously described by the senior author (R. W.-W. H.) were routinely taken. ${ }^{[29]}$ The lower extremities were fully extended and positioned so that the tibial tuberosities were facing forward and the lateral malleoli were $30 \mathrm{~cm}$ apart. This standard position ensures that the tibia were vertical and facing forward with minimal rotation. The center of the femoral head $(\mathrm{H})$ was determined using Mose's circles; the knee center $(\mathrm{K})$ is the midpoint of the tibial spines; the ankle center $(\mathrm{A})$ is the midpoint between the inner edges of the malleoli and one-half of the height of the talus. The femoral length is defined as the distance from the superior dome of the femoral head to the inferior surface of the medial femoral condyle. The midpoint of femoral neck base was designated as $\mathrm{N}$; $\mathrm{HN}$ (bisector of femoral neck) is the femoral neck axis connecting the center of the femoral head and the midpoint of the femoral neck. The femoral diaphysis was divided into three equal parts, and four points were marked on the femoral shaft as follows: The midpoint of cortical width at lesser trochanter was designated as $\mathrm{Fs}$; a point bisecting the shaft $10 \mathrm{~cm}$ proximal to the knee joint was designated as $\mathrm{F} d$; the femoral shaft was defined as $\mathrm{Fs} \mathrm{F} d$; and the proximal and distal one-third bicortical centers for the femur were designated as $\mathrm{F} p$ and $\mathrm{Fm}$, respectively. A point bisecting the shaft midway between $\mathrm{Fs}$ and $\mathrm{F} d$ was designated as $\mathrm{F} c$. The proximal femoral anatomic axis was defined as FsFp. B is the intersection of the femoral neck axis and the proximal femoral anatomic axis [Figures 1 and 2]. ${ }^{[2,29]}$

The line connecting the center of the femoral head to the center of the knee joint was used as the femoral mechanical axis (HK) and the line connecting the center of the knee joint to the center of the ankle joint was used as the tibial mechanical axis (KA). The mechanical axes of the lower limb were defined as the angle between femoral mechanical axis and tibial mechanical axis (HK-KA). For measurement of the valgus correction angle of the distal femur, the line connecting the distal one-third bicortical centers to the center of the knee was used as the anatomical axis of the distal femur $(\mathrm{F} m \mathrm{~K})$. This is the expected pathway of the IM alignment rod in the distal femur. The femoral neck-shaft angle was defined as the angle between the femoral neck axis and the proximal femoral anatomic axis (HB-BFp). The valgus correction angle of the distal femur was defined as the angulation between the femoral mechanical axis and the distal anatomical axis of the femur (HK-FmK). ${ }^{[29]}$ The percentage of patients with distal femoral valgus resection angle outside the range of $5^{\circ} \pm 2^{\circ}$ was determined. ${ }^{[29,30]}$ The magnitude of coronal femoral bowing angle $(\mathrm{F} s \mathrm{~F} c-\mathrm{F} c \mathrm{~F} d)$ was measured using the method described by Mullaji et al. ${ }^{[2]}$ 


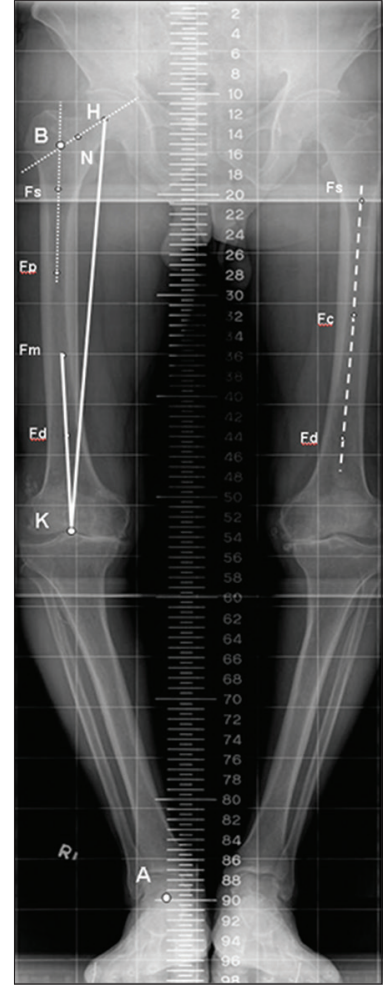

Figure 1: A schematic diagram illustrating the key radiographic landmarks used to define the axial alignment parameters. $\mathrm{H}$, femoral head center; $\mathrm{N}$, midpoint of femoral neck base; Fs, midpoint of cortical width at lesser trochanter; Fp, midpoint of cortical width at proximal one-third femoral length; $\mathrm{HN}$, bisector of femoral neck; FsFp, anatomic axis of proximal femur; B, intersection of lines HN and FsFp; Fm, midpoint of distal one-third femoral length; Fd, a point bisecting the shaft $10 \mathrm{~cm}$ proximal to the knee joint; K, knee joint center; a point bisecting the shaft midway between Fs and Fd was designated as Fc; HK, mechanical axis of femur; A, ankle joint center; KA, mechanical axis of tibia; HK-FmK, the valgus correction angle of the distal femur; HB-BFp, the femoral neck-shaft angle; $\mathrm{FsFc}-\mathrm{FcFd}$, the coronal femoral bowing angle.

According to Mullaji's criteria, ${ }^{[26]}$ patients with a coronal femoral bowing angle $>5^{\circ}$ were defined as having excessive coronal femoral bowing deformity [Figures 1 and 2].

Clinical data including age, gender, body weight, body height, mean body mass index, and radiographic parameters including femoral length, preoperative mechanical axes, femoral neck-shaft angle, the coronal femoral bowing angle, and the distal femoral valgus resection angle were recorded. All data were collected and entered in an Excel spreadsheet (Microsoft, Redmond, WA, USA) by two independent researchers. The data were rechecked for missing and illogical data, and subsequently copied into SPSS version 13.0 (SPSS Inc., Chicago, IL, USA) and statistical analyses were performed. For categorical data, Chi-square analysis or the Fisher exact test was used as appropriate. For numerical data, a two-tailed Student's $t$-test was used for comparisons between the two groups.

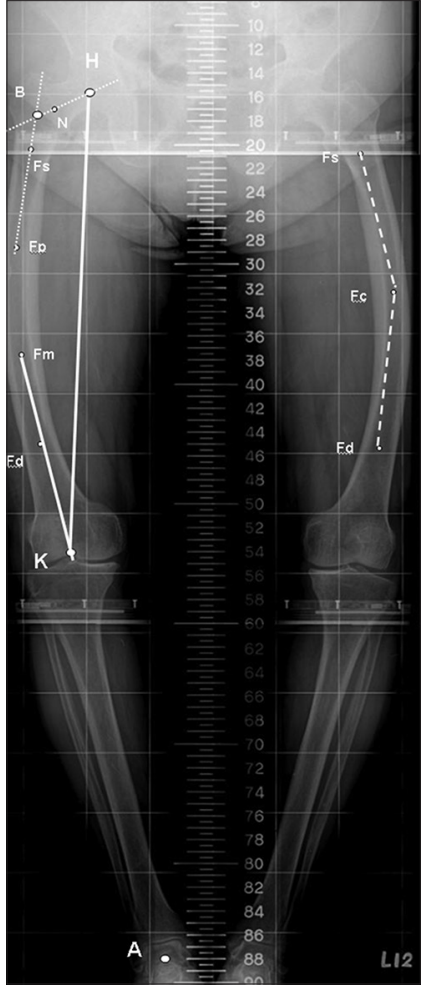

Figure 2: Preoperative long-leg weight-bearing split scanogram showing excessive coronal femoral bowing deformity. The distal femoral valgus resection angle was $12^{\circ}$, the femoral neck-shaft angle was $131^{\circ}$, and the coronal femoral bowing angle was 10 .

Statistical analysis was conducted by an independent statistician. A value of $p<0.05$ was considered statistically significant. Correlation coefficients were graded using previously described semi-quantitative criteria: Excellent for $0.9 \leq r \leq 1.0$, good for $0.7 \leq r \leq 0.89$, fair/moderate for $0.5 \leq r \leq 0.69$, low for $0.25 \leq r \leq 0.49$, and poor for $0.0 \leq r \leq 0.24 .^{[31]}$

\section{RESULTS}

A total of 526 patients (952 knees) with end-stage osteoarthritic knees in conjunction with genu varum deformity who underwent primary TKA were enrolled in this study. There were 152 males (248 knees) and $374 \mathrm{fe}$ males (704 knees) with a mean age of 72 years (range, 59-82 years) at the time of surgery. The mean body height was $154 \mathrm{~cm}$ (range, 141-177 cm), the mean body weight $66 \mathrm{~kg}$ (range, $38-97 \mathrm{~kg}$ ), and the mean body mass index was $27.7 \mathrm{~kg} / \mathrm{m}^{2}$ (range, $17.6-36.2 \mathrm{~kg} / \mathrm{m}^{2}$ ). Computer measurements of digital films showed the following: Mean femoral length $454 \mathrm{~mm}$ (range, 412-524 $\mathrm{mm}$ ), mean preoperative mechanical axis $166^{\circ}\left(\right.$ range, $\left.146^{\circ}-173^{\circ}\right)$, mean distal femoral valgus resection angle $7^{\circ}$ (range, $4^{\circ}-14^{\circ}$ ), mean coronal femoral bowing angle $4^{\circ}$ (range, $1^{\circ}-19^{\circ}$ ), and mean femoral neck-shaft angle $130^{\circ}\left(\right.$ range, $\left.116^{\circ}-145^{\circ}\right)$ [Table 1]. 
Three hundred and four (31.9\%) of 952 knees in this cohort had a distal femoral valgus resection angle value outside the commonly used reference range of $5^{\circ} \pm 2^{\circ}$. There was a statistically significant difference in the mean distal femoral valgus resection angle between the male versus female $\left[5^{\circ}\left(\right.\right.$ range, $\left.4^{\circ}-10^{\circ}\right)$ vs. $8^{\circ}\left(\right.$ range, $\left.\left.5^{\circ}-14^{\circ}\right) ; p<0.001\right]$ and non-bowed femur versus bowed femur $\left[7^{\circ}\right.$ (range, $4^{\circ}-10^{\circ}$ ) vs. $10^{\circ}$ (range, $\left.\left.8^{\circ}-14^{\circ}\right) ; p<0.001\right]$ cohorts. With regard to the correlation coefficients between the distal femoral valgus resection angle and the other radiographic measurements made, only the coronal femoral bowing angle demonstrated a good correlation $(r=0.72)$. Low correlation coefficients with the distal femoral valgus resection angle were found with body height, body weight, femoral neck-shaft angle, and preoperative mechanical axis $(r=$ $-0.25,-0.27,-0.28$, and 0.41 , respectively). The remaining variables including the patient's age, body mass index, and femoral length showed poor correlation coefficients with the distal femoral valgus resection angle $(r=0.05,-0.09$, and -0.19 , respectively). In patients with more coronal femoral bowing angle, the distal femoral valgus resection angle had a tendency to increase [Table 2].

Patients were further divided into two groups: Those without excessive coronal femoral bowing deformity were assigned to group A and those with excessive coronal femoral bowing deformity (greater than $5^{\circ}$ as defined by Mullaji's criteria) were in group B. There were 859 knees in group A and 93 knees in group B. Demographically, significant differences were revealed in terms of gender, age at the time of operation, femoral length, femoral neck-shaft angle, the distal femoral valgus resection angle, the coronal femoral bowing angle, and preoperative mechanical axes between the non-bowed and bowed femur. The majority of coronal femoral bowing deformity occurs in elderly female Asian patients [Table 3].

\section{DISCUSSION}

The key finding in this investigation was that the distal femoral valgus resection angle has high variability and is affected by the excessive coronal femoral bowing deformity found in elderly female Asian patients who do not have a history of past trauma or surgery. The $5^{\circ}-6^{\circ}$ distal femoral valgus resection angle does not hold true in the ethnic Asian population.

Three hundred and four (31.9\%) of 952 knees had a value outside the commonly accepted reference range of $5^{\circ} \pm 2^{\circ}$. In two previous studies, it was stated that taller patients would have a smaller distal femoral valgus resection angle, and that the distal femoral valgus resection angle is related to the femoral neck-shaft angle in male patients. ${ }^{[15,21]}$ Our data do not support these findings. Only the coronal femoral bowing deformity seems to be a significant factor and has a direct correlation with the distal femoral valgus
Table 1: Patient demographic data and preoperative radiographic data

\begin{tabular}{lc}
\hline Parameters & Overall $(N=952)$ \\
\hline Demographic variables & \\
Age (years) & $72 \pm 6(59-86)$ \\
Gender & \\
$\quad$ Male & $248(26.1)$ \\
Female & $704(73.9)$ \\
Body height $(\mathrm{cm})$ & $154 \pm 8(138-172)$ \\
Body weight $(\mathrm{kg})$ & $66 \pm 11(38-96)$ \\
Body mass index $\left(\mathrm{kg} / \mathrm{m}^{2}\right)$ & $27.6 \pm 4(17.6-37.7)$ \\
Radiographic measurements & \\
Femoral length $(\mathrm{mm})$ & $454 \pm 30(412-524)$ \\
Mechanical axis $\left({ }^{\circ}\right)$ & $166 \pm 6(146-173)$ \\
Distal femoral valgus resection angle $\left(^{\circ}\right)$ & $7 \pm 2(4-14)$ \\
Coronal femoral bowing angle $\left(^{\circ}\right)$ & $4 \pm 3(1-19)$ \\
Femoral neck-shaft angle $\left({ }^{\circ}\right)$ & $130 \pm 5(116-145)$ \\
\hline
\end{tabular}

The values are presented as the mean \pm SD with the range in parentheses, or $n(\%)$ where appropriate

Table 2: Calculated correlation coefficients of the distal femoral valgus resection angle and both demographic variables and preoperative radiographic measurements

\begin{tabular}{lcl}
\hline Variable & $\begin{array}{c}\text { Correlation coefficient } \\
\text { with distal femoral } \\
\text { valgus resection angle }\end{array}$ & Grade \\
\hline Demographic variables & 0.05 & Poor \\
Age (years) & -0.25 & Low \\
Body height $(\mathrm{cm})$ & -0.27 & Low \\
Body weight $(\mathrm{kg})$ & -0.09 & Poor \\
Body mass index $(\mathrm{kg} / \mathrm{m} 2)$ & & \\
Radiographic measurements & -0.19 & Poor \\
Femoral length $(\mathrm{mm})$ & 0.41 & Low \\
Mechanical axis $\left({ }^{\circ}\right)$ & 0.72 & Good \\
Coronal femoral bowing angle $\left(^{\circ}\right)$ & -0.28 & Low \\
Femoral neck-shaft angle $\left(^{\circ}\right)$ &
\end{tabular}

A negative value corresponds with an inverse relationship

resection angle.

In this study, it was found that the majority of coronal femoral bowing deformity occurs in elderly female Asian patients. It has been noted that there is a relatively high prevalence of coronal femoral bowing in this population. ${ }^{[12,25-27]}$ Mullaji suggested it may result from a combination of osteomalacia and osteoporosis caused by nutritional deficiency of vitamin D and calcium. ${ }^{[26]}$ Because bowing may not be apparent either clinically or on plain radiographs of the knee, the extent of coronal femoral bowing deformity is likely underestimated in clinical practice unless preoperative long-leg weight-bearing split scanograms are routinely obtained. A long-leg weight-bearing split scanogram should be preoperatively obtained to determine the true distal femoral valgus resection angle in elderly female Asian patients. In 
Table 3: Demographic and radiographic data in patients of the non-bowed and bowed groups

\begin{tabular}{|c|c|c|c|}
\hline Parameters & Group A $(n=859)$ & Group B $(n=93)$ & $p$ \\
\hline \multicolumn{4}{|l|}{ Demographic variables } \\
\hline Age (years) & $72 \pm 6(59-86)$ & $75 \pm 5(64-82)$ & $<0.001 *$ \\
\hline$<70$ & $246(28.6)$ & $12(12.9)$ & $0.001 *$ \\
\hline $70-80$ & $553(64.4)$ & $57(61.3)$ & 0.571 \\
\hline$>80$ & $60(7.0)$ & $24(25.8)$ & $<0.001 *$ \\
\hline \multicolumn{4}{|l|}{ Gender } \\
\hline Male & $246(28.6)$ & $2(2.2)$ & $<0.001 *$ \\
\hline Female & $613(71.4)$ & $91(97.8)$ & $<0.001 *$ \\
\hline Body height $(\mathrm{cm})$ & $154 \pm 8(138-172)$ & $151 \pm 8(140-164)$ & $<0.001 *$ \\
\hline Body weight $(\mathrm{kg})$ & $66 \pm 11(38-96)$ & $62 \pm 8(42-82)$ & $<0.001 *$ \\
\hline Body mass index $\left(\mathrm{kg} / \mathrm{m}^{2}\right)$ & $27.7 \pm 4(17.6-37.7)$ & $27.2 \pm 4(18.4-35.9)$ & 0.256 \\
\hline \multicolumn{4}{|l|}{ Radiographic measurements } \\
\hline Femoral length (mm) & $456 \pm 30(412-524)$ & $440 \pm 22(412-520)$ & $<0.001 *$ \\
\hline Mechanical axis $\left(^{\circ}\right)$ & $167 \pm 6(146-173)$ & $164 \pm 4(156-173)$ & $0.004 *$ \\
\hline Distal femoral valgus resection angle $\left(^{\circ}\right)$ & $7 \pm 1(4-10)$ & $10 \pm 1(8-14)$ & $<0.001 *$ \\
\hline Coronal femoral bowing angle $\left(^{\circ}\right)$ & $3 \pm 1(1-4)$ & $11 \pm 3(8-19)$ & $<0.001 *$ \\
\hline Femoral neck-shaft angle $\left(^{\circ}\right)$ & $131 \pm 4(118-142)$ & $128 \pm 5(116-145)$ & $0.006^{*}$ \\
\hline
\end{tabular}

*Statistically significant $(p<0.05)$. Group A: Patients without excessive coronal femoral bowing deformity; Group B: Patients with excessive coronal femoral bowing deformity. The values are presented as mean $\pm \mathrm{SD}$ with the range in parentheses, or $n(\%)$ where appropriate.

this series, the mean distal femoral valgus resection angle was $10^{\circ} \pm 1^{\circ}$. The majority of currently available IM or extramedullary alignment guidance system femoral jigs do not provide a wide enough choice of valgus resection angle to accommodate such magnitude of deformity. If a fixed distal femoral valgus resection angle of $6^{\circ}$ is used, the unexpected greater distal femoral valgus resection angle would result in greater risk of malposition of components, leading to inadequate postoperative mechanical axes and aseptic failure of TKA. ${ }^{[1-14,18,25-27,32,33]}$

Femoral component malalignment is not uncommon when using an IM alignment guide. ${ }^{[11-17]}$ Mason et al. performed a meta-analysis of 29 studies comparing computer-assisted surgery-TKA (CAS-TKA) to conventional TKA. ${ }^{[33]}$ They found that $90.4 \%$ of CAS-TKAs had a femoral component alignment within $2^{\circ}$ perpendicular to the mechanical axis versus only $65.9 \%$ using conventional techniques. The CT-free navigation system (Brain-LAB, Inc., Munich, Germany) has been available at the Chiayi Chang Gung Memorial Hospital since 2002. Our previous studies demonstrated that the CAS-TKA improved the accuracy of the orientation of components and mechanical axis of the lower limb. ${ }^{[11-14]}$ CAS-TKA also has been shown to improve component alignments, limb axis correction, and soft tissue balance in patients with arthritic knees complicated by extra-articular deformity. ${ }^{[27]}$ Use of CAS-TKA that aligns the reconstructed mechanical axis based on the hip and knee centers (thereby ignoring extra-articular deformity such as coronal femoral bowing) makes the need for preoperative long-leg weight-bearing split scanograms for planning distal femoral resection angle almost redundant. This technol- ogy is used in few centers, and thus, preoperative long-leg weight-bearing split scanograms are important where this technology is not available.

Several limitations in this study must be acknowledged. This was a radiological study in which we were unable to assess any correlation between alignment and functional outcome. The study was retrospective in design, but all the patients underwent TKA following the same protocol in a single center with a registry system, which might reduce the inherent bias. By design, the current study was limited to knee replacements in ethnic Asian patients, so it not applicable to other ethnicities. While we have confirmed the high incidence of coronal femoral bowing deformity in elderly female ethnic Asians, we do not have an explanation why this occurs.

The authors wish to call attention to their finding that $32 \%$ of ethnic Asians patients who have end-stage osteoarthritis of the knee joint and genu varum deformity will have a distal femoral valgus resection angle that is outside the range of $5^{\circ} \pm 2^{\circ}$. Although whether a long-leg weight-bearing split scanogram should be a routine part of the preoperative protocol for TKA is still debated, ${ }^{[3,25,26]}$ our protocol calls for taking a long-leg weight-bearing split scanogram to determine the true distal femoral valgus resection angle in an ethnic Asian population. Special jigs that allow a wide enough choice of valgus cut angle, simultaneous or staged corrective osteotomy combined with TKA, ${ }^{[22]}$ or intra-articular bone resection technique ${ }^{[23]}$ can then be used to accommodate the deformity and provide an ideal reconstructed mechanical axis and accurate placement of components. 


\section{REFERENCES}

1. Bargren JH, Blaha JD, Freeman MA. Alignment in total knee arthroplasty. Correlated biomechanical and clinical observations. Clin Orthop Relat Res 1983;173:178-83.

2. Insall JN, Binazzi R, Soudry M, Mestriner LA. Total knee arthroplasty. Clin Orthop Relat Res 1985;192:13-22.

3. Jeffery RS, Morris RW, Denham RA. Coronal alignment after total knee replacement. J Bone Joint Surg Br 1991;73:709-14.

4. Ritter MA, Faris PM, Keating EM, Meding JB. Postoperative alignment of total knee replacement. Its effect on survival. Clin Orthop Relat Res 1994;299:153-6.

5. Deakin AH, Basanagoudar PL, Nunag P, Johnston AT, Sarungi M. Natural distribution of the femoral mechanical-anatomical angle in an osteoarthritic population and its relevance to total knee arthroplasty. Knee 2012;192:120-3.

6. Fang DM, Ritter MA, Davis KE. Coronal alignment in total knee arthroplasty: Just how important is it? J Arthroplasty 2009;24 (6 Suppl):39-43.

7. Parratte S, Pagnano MW, Trousdale RT, Berry DJ. Effect of postoperative mechanical axis alignment on the fifteen-year survival of modern, cemented total knee replacements. J Bone Joint Surg Am 2010;92:2143-9.

8. Vessely MB, Whaley AL, Harmsen WS, Schleck CD, Berry DJ. Long-term survivorship and failure modes of 1000 cemented condylar total knee arthroplasties. Clin Orthop 2006;452:28-34.

9. Chen JH, Kuo FC, Wang JW. Total knee arthroplasty in patients with dialysis: Early complications and mortality. Biomed J 2014;37:84-9.

10. Taylor M, Barrett DS. Explicit finite element simulation of eccentric loading in total knee replacement. Clin Orthop Relat Res 2003;414:162-71

11. Huang TW, Hsu WH, Peng KT, Hsu RW. Total knee replacement in patients with significant femoral bowing in the coronal plane: A comparison of conventional and computer-assisted surgery in an Asian population. J Bone Joint Surg Br 2011;93:345-50.

12. Huang TW, Hsu WH, Peng KT, Hsu RW, Weng YJ, Shen WJ. Total knee arthroplasty with use of computer-assisted navigation compared with conventional guiding systems in the same patient: Radiographic results in Asian patients. J Bone Joint Surg Am 2011;93:1197-202.

13. Kuo LT, Huang TW, Peng KT, Hsu RW. Computer-assisted navigation for cruciate-retaining total knee arthroplasty in patients with advanced valgus arthritic knees. Formos J Musculoskelet Disord 2013;4:99-104.

14. Huang TW, Kuo LT, Peng KT, Lee MS, Hsu RW. Computed Tomography Evaluation in Total Knee Arthroplasty: Computer-Assisted Navigation Versus Conventional Instrumentation in Patients With Advanced Valgus Arthritic Knees. J Arthroplasty 2014;29:2363-8

15. Bardakos N, Cil A, Thompson B, Stocks G. Mechanical axis cannot be restored in total knee arthroplasty with a fixed valgus resection angle: A radiographic study. J Arthroplasty 2007;22 (6 Suppl 2):85-9.

16. Mahaluxmivala J, Bankes MJ, Nicolai P, Aldam CH, Allen PW. The effect of surgeon experience on component positioning in 673 Press Fit Condylar posterior cruciate-sacrificing total knee arthroplasties.
J Arthroplasty 2001;16:635-40.

17. Petersen TL, Engh GA. Radiographic assessment of knee alignment after total knee arthroplasty. J Arthroplasty 1988;3:67-72.

18. Stulberg SD. How accurate is current TKR instrumentation? Clin Orthop Relat Res 2003;416:177-84.

19. Bankes MJ, Back DL, Cannon SR, Briggs TW. The effect of component malalignment on the clinical and radiological outcome of the Kinemax total knee replacement. Knee 2003;10:55-60.

20. Ritter MA, Davis KE, Meding JB, Pierson JL, Berend ME, Malinzak RA. The effect of alignment and BMI on failure of total knee replacement. J Bone Joint Surg Am 2011;93:1588-96.

21. Canale ST, Beaty JH: Campbell's operative orthopaedics. $11^{\text {th }}$ ed.. Philadelphia, PA: Elsevier Inc.; 2008.

22. Lonner JH, Siliski JM, Lotke PA. Simultaneous femoral osteotomy and total knee arthroplasty for treatment of osteoarthritis associated with severe extra-articular deformity. J Bone Joint Surg Am $2000 ; 82: 342-8$

23. Wang JW, Wang CJ. Total knee arthroplasty for arthritis of the knee with extra-articular deformity. J Bone Joint Surg Am 2002;84:1769-74.

24. Ko PS, Tio MK, Ban CM, Mak YK, Ip FK, Lam JJ. Radiologic analysis of the tibial intramedullary canal in Chinese varus knees: Implications in total knee arthroplasty. J Arthroplasty $2001 ; 16: 212-5$

25. Yau WP, Chiu KY, Tang WM, Ng TP. Coronal bowing of the femur and tibia in Chinese: Its incidence and effects on total knee arthroplasty planning. J Orthop Surg (Hong Kong) 2007;15:32-6.

26. Mullaji AB, Marawar SV, Mittal V. A comparison of coronal plane axial femoral relationships in Asian patients with varus osteoarthritic knees and healthy knees. J Arthroplasty 2009;24:861-7.

27. Mullaji A, Shetty GM. Computer-assisted total knee arthroplasty for arthritis with extra-articular deformity. J Arthroplasty 2009;24:1164-9.e1.

28. Nam D, Maher PA, Robles A, McLawhorn AS, Mayman DJ. Variability in the relationship between the distal femoral mechanical and anatomical axes in patients undergoing primary total knee arthroplasty. J Arthroplasty 2013;28:798-801.

29. Hsu RW, Himeno S, Coventry MB, Chao EY. Normal axial alignment of the lower extremity and load-bearing distribution at the knee. Clin Orthop Relat Res 1990;255:215-27.

30. Wang Y, Zeng Y, Dai K, Zhu Z, Xie L. Normal lower-extremity alignment parameters in healthy Southern Chinese adults as a guide in total knee arthroplasty. J Arthroplasty 2010;25:563-70.

31. Munro BH. Correlation. Statistical Methods for Healthcare Research. $3^{\text {rd }}$ ed. USA: Lippincott-Raven; 1997.

32. Sharkey PF, Hozack WJ, Rothman RH, Shastri S, Jacoby SM. Insall Award paper. Why are total knee arthroplasties failing today? Clin Orthop Relat Res 2002;404:7-13.

33. Mason JB, Fehring TK, Estok R, Banel D, Fahrbach K. Meta-analysis of alignment outcomes in computer-assisted total knee arthroplasty surgery. J Arthroplasty 2007;22:1097-106. 